\title{
Generalized Homogeneous Polynomials for Efficient Template-Based Nonlinear Invariant Synthesis
}

\author{
Kensuke Kojima $^{1,2}$, Minoru Kinoshita ${ }^{1,4}$, and Kohei Suenaga ${ }^{1,3}$ \\ 1 Kyoto University \\ 2 JST CREST \\ 3 JST PRESTO \\ ${ }^{4}$ KLab Inc.
}

\begin{abstract}
The template-based method is one of the most successful approaches to algebraic invariant synthesis. In this method, an algorithm designates a template polynomial $p$ over program variables, generates constraints for $p=0$ to be an invariant, and solves the generated constraints. However, this approach often suffers from an increasing template size if the degree of a template polynomial is too high.

We propose a technique to make template-based methods more efficient. Our technique is based on the following finding: If an algebraic invariant exists, then there is a specific algebraic invariant that we call a generalized homogeneous algebraic invariant that is often smaller. This finding justifies using only a smaller template that corresponds to a generalized homogeneous algebraic invariant.

Concretely, we state our finding above formally based on the abstract semantics of an imperative program proposed by Cachera et al. Then, we modify their template-based invariant synthesis so that it generates only generalized homogeneous algebraic invariants. This modification is proved to be sound. Furthermore, we also empirically demonstrate the merit of the restriction to generalized homogeneous algebraic invariants. Our implementation outperforms that of Cachera et al. for programs that require a higher-degree template.
\end{abstract}

\section{Introduction}

We consider the following postcondition problem: Given a program $c$, discover a fact that holds at the end of $c$ regardless of the initial state. This paper focuses on a postcondition written as an algebraic condition $p_{1}=0 \wedge \cdots \wedge p_{n}=0$, where $p_{1}, \ldots, p_{n}$ are polynomials over program variables; this problem is a basis for static verification of functional correctness.

One approach to this problem is invariant synthesis, in which we are to compute a family of predicates $P_{l}$ indexed by program locations $l$ such that $P_{l}$ holds whenever the execution of $c$ reaches $l$. The invariant associated with the end of $c$ is a solution to the postcondition problem. 
Because of its importance in static program verification, algebraic invariant synthesis has been intensively studied [4, 16, 19,20]. Among these proposed techniques, one successful approach is the constraint-based method in which invariant synthesis is reduced to a constraint-solving problem. During constraint generation, this method designates templates, which are polynomials over the program variables with unknown parameters at the coefficient positions 20. The algorithm generates constraints that ensure that the templates are invariants and obtains the invariants by solving the constraint: 5 .

Example 1. The program $c_{\text {fall }}$ in Figure 1 models the behavior of a mass point with weight 1 and with a constant acceleration rate; the program takes friction between the mass point and air into account 6 . For this program, the postcondition $-g t+g t_{0}-v+v_{0}-x \rho+x_{0} \rho=0$ holds regardless of the initial state.

We describe how a template-based method computes the postcondition in Example 1. The method described here differs from the one we explore in this paper; this explanation is intended to suggest the flavor of a template method.

A template-based method generates a template polynomial over the program variables that represent an invariant at Line 4 .

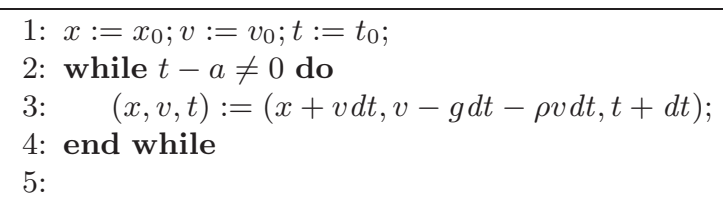

Fig. 1. Program $c_{\text {fall }}$, which models a falling mass point. The symbols in the program represent the following quantities: $x$ is the position of the point, $v$ is its speed, $t$ is time, $x_{0}$ is the initial position, $v_{0}$ is the initial speed, $t_{0}$ is the initial value of the clock $t, g$ is the acceleration rate, $\rho$ is the friction coefficient, and $d t$ is the discretization interval. The simultaneous substitution in the loop body numerically updates the values of $x, v$, and $t$. The values of $x, v$, and $t$ are numerical solutions of the differential equations $\frac{d x}{d t}=v$ and $\frac{d t}{d t}=1$; notice that the force applied by the air to the mass point is $-\rho v$, which leads to the differential equation for $\frac{d v}{d t}=-g-\rho v$.

Suppose the generated polynomial $p\left(x_{0}\right.$, $\left.v_{0}, t_{0}, x, v, t, a, d t, g, \rho\right)$ is of degree 2 over the variables: $p\left(x_{0}, v_{0}, t_{0}, x, v, t, a, d t\right.$, $g, \rho):=a_{1}+a_{t_{0}} t_{0}+a_{x_{0}} x_{0}+\cdots+a_{g \rho} g \rho$, where $a_{w}$ is the coefficient parameter associated with the power product $w$. The procedure then generates constraints such that $p\left(x_{0}, v_{0}, t_{0}, x, v, t, a, d t, g, \rho\right)=0$ is indeed an invariant at Line 4. The method proposed by Sankaranarayanan et al. 20, based on the Gröbner basis [5] generates the constraints as an equations over the parameters; in this case, a solution to the constraints gives $-g t+g a-v+v_{0}-x \rho+x_{0} \rho=0$, which is indeed an invariant at the end of $c_{\text {fall }}$.

\footnotetext{
${ }^{5}$ The constraint-based method by Cachera et al. 4], which is the basis of the current paper, uses a template also for other purposes. See Section 6 for details.

${ }^{6}$ Although the guard condition $t-a \neq 0$ should be $t-a<0$ in a real-world numerical program, we use the current example for presentation purposes.
} 
One of the drawbacks of the template-based method is excessive growth of the size of a template. Blindly generating a template of degree $d$ for a degree parameter $d$ makes the invariant synthesis less scalable for higher-degree invariants. For example, the program in Example 1 has an invariant $-g t^{2}+g t_{0}^{2}-$ $2 t v+2 t_{0} v_{0}+2 x-2 x_{0}=0$ at Line 4 . This invariant requires a degree- 3 template, which has $\left(\begin{array}{c}10+3 \\ 3\end{array}\right)=286$ monomials in this case.

We propose a hack to alleviate this drawback in the template-based methods. Our method is inspired by a rule of thumb in physics called the principle of quantity dimension: A physical law should not add two quantities with different quantity dimensions 2 . If we accept this principle, then, at least for a physically meaningful program such as $c_{f a l l}$, an invariant (and therefore a template) should consist of monomials with the same quantity dimensions.

Indeed, the polynomial $-g t+g t_{0}-v+v_{0}-x \rho+x_{0} \rho$ in the invariant calculated in Example 1 consists only of quantities that represent velocities. (Notice that $\rho$ is a quantity that corresponds to the inverse of a time quantity.) The polynomial $-g t^{2}+g t_{0}^{2}-2 t v+2 t_{0} v_{0}+2 x-2 x_{0}$ above consists only of quantities corresponding to the length. If we use the notation of quantity dimensions used in physics, the former polynomial consists only of monomials with the quantity dimension $L T^{-1}$, whereas the latter consists only of $L$, where $L$ and $T$ represent quantity dimensions for lengths and times, respectively.

By leveraging the quantity dimension principle in the template synthesis phase, we can reduce the size of a template. For example, we could use a template that consists only of monomials for, say, velocity quantities instead of the general degree-2 polynomial $p\left(x_{0}, v_{0}, x, v, t, a, d t, g, \rho\right)$ used above, which yields a smaller template.

The idea of the quantity dimension principle can be nicely captured by generalizing the notion of homogeneous polynomials. A polynomial is said to be homogeneous if it consists of monomials of the same degree; for example, the polynomial $x^{3}+x^{2} y+x y^{2}+y^{3}$ is a homogeneous polynomial of degree 3 . We generalize this notion of homogeneity so that (1) a degree is an expression corresponding to a quantity dimension (e.g., $L T^{-1}$ ) and (2) each variable has its own degree in degree computation.

Let us describe our idea using an example, deferring formal definitions. Suppose we have the following degree assignment for each program variable: $\Gamma:=$ $\left\{x_{0} \mapsto L, t_{0} \mapsto T, g \mapsto L T^{-2}, t \mapsto T, d t \mapsto T, x \mapsto L, v \mapsto L T^{-1}, v_{0} \mapsto L T^{-1}\right.$, $\left.\rho \mapsto T^{-1}, a \mapsto T\right\}$. This degree assignment intuitively corresponds to the assignment of the quantity dimension to each variable. With this degree assignment $\Gamma$, all of the monomials in $-g t+g t_{0}-v+v_{0}-x \rho+x_{0} \rho$ have the same degree; for example, the monomial $-g t$ has degree $\Gamma(g) \Gamma(t)=\left(L T^{-2}\right) T=L T^{-1}$ and monomial $x \rho$ has degree $\Gamma(x) \Gamma(\rho)=L T^{-1}$, and so on. Hence, $-g t+g t_{0}-v+v_{0}-x \rho+x_{0} \rho$ is a homogeneous polynomial in the generalized sense. Such a polynomial is called a generalized homogeneous (GH) polynomial. We call an algebraic invariant with a GH polynomial a generalized homogeneous algebraic (GHA) invariant.

The main result of this paper is a formalization of this idea: If there is an algebraic invariant of a given program $c$, then there is a GHA invariant. 
This justifies the use of a template that corresponds to a GH polynomial in the template method. We demonstrate this result by using the abstract semantics of an imperative programming language proposed by Cachera et al. [4. We also empirically show that the algorithm by Cachera et al. can be made more efficient using this idea.

As we saw above, the definition of GH polynomials is parameterized over a degree assignment $\Gamma$. The type inference algorithm for the dimension type system proposed by Kennedy [12,13] can be used to find an appropriate degree assignment; $\Gamma$ above is inferred using this algorithm. The dimension type system was originally proposed for detecting a violation of the quantity-dimension principle in a numerical program. Our work gives an application of the dimension type system to invariant synthesis.

Although the method is inspired by the principle of quantity dimensions, it can be applied to a program that does not model a physical phenomenon because we abstract the notion of a quantity dimension using that of generalized homogeneity. All the programs used in our experiments (Section 7) are indeed physically nonsensical programs.

The rest of this paper is organized as follows. Section 2 sets up the basic mathematical definitions used in this paper; Section 3 defines the syntax and semantics of the target language and its abstract semantics; Section 4 defines GH polynomials; Section 5 defines the revised abstract semantics as the restriction of the original one to the set of GH polynomials and shows that the revised semantics is sound and complete; Section [ gives a template-based invariantsynthesis algorithm and shows its soundness; Section 7 reports the experimental results; Section 8 discusses related work; and Section 9 presents the conclusions. Several proofs are given in the appendices .

\section{Preliminaries}

$\mathbb{R}$ is the set of real numbers and $\mathbb{N}$ is the set of natural numbers. We write $|S|$ for the cardinality of $S$ if $S$ is a finite set. We designate an infinite set of variables Var. $K$ is a field ranged over by metavariable $k$; we use the standard notation for the operations on $K$. For $x_{1}, \ldots, x_{n} \in$ Var, we write $K\left[x_{1}, \ldots, x_{n}\right]$, ranged over by $p$ and $q$, for the set of polynomials in $x_{1}, \ldots, x_{n}$ over $K$.

A subset $I \subseteq K\left[x_{1}, \ldots, x_{n}\right]$ is called an ideal if (1) $I$ is an additive subgroup and (2) $p q \in I$ for any $p \in I$ and $q \in K\left[x_{1}, \ldots, x_{n}\right]$. A set $S \subseteq K\left[x_{1}, \ldots, x_{n}\right]$ is said to generate the ideal $I$, written $I=\langle S\rangle$, if $I$ is the smallest ideal that contains $S$.

We call an expression of the form $x_{1}^{d_{1}} \ldots x_{N}^{d_{N}}$, where $d_{1}, \ldots, d_{N} \in \mathbb{N}$ and $x_{1}$, $\ldots, x_{N} \in$ Var, a power product over $x_{1}, \ldots, x_{n} ; w$ is a metavariable for power products. We call $\sum d_{i}$ the degree of this power product. A monomial is a term of the form $k w$; the degree of this monomial is that of $w$. We write $\operatorname{deg}(p)$, the degree of the polynomial $p$, for the maximum degree of the monomials in $p$.

A state, ranged over by $\sigma$, is a finite map from Var to $K$. We write $\mathbf{S t}$ for the set of states. We use the metavariable $S$ for a subset of St. We write $\sigma(p)$ for 
the evaluated value of $p$ under $\sigma$. Concretely, $\sigma(p):=p\left(\sigma\left(x_{1}\right), \ldots, \sigma\left(x_{n}\right)\right)$. The set $\mathcal{P}(\mathbf{S t})$ constitutes a complete lattice with respect to the set-inclusion order.

\section{Language}

This section defines the target language, its concrete semantics, and its abstract semantics. We essentially follow the development by Cachera et al. 4]; we refer the interested reader to this paper.

The syntax of the target language is as follows:

$c::=\operatorname{skip}|x:=p| c_{1} ; c_{2} \mid$ if $p=0$ then $c_{1}$ else $c_{2} \mid$ while $p=0$ do $c \mid$ while $p \neq 0$ do $c$

where $p$ is a polynomial over the program variables. We restrict the guard to a single-polynomial algebraic condition (i.e., $p=0$ ) or its negation.

The semantics of this language is given by the following denotation function, which is essentially the same as that by Cachera et al.

$$
\begin{aligned}
\llbracket c \rrbracket & :(\mathcal{P}(\mathbf{S t}), \subseteq) \rightarrow(\mathcal{P}(\mathbf{S t}), \subseteq) \\
\llbracket \text { skip } \rrbracket(S) & =S \\
\llbracket x:=p \rrbracket(S) & =\{\sigma \mid \sigma[x \mapsto \sigma(p)] \in S\} \\
\llbracket c_{1} ; c_{2} \rrbracket(S) & =\llbracket c_{1} \rrbracket\left(\llbracket c_{2} \rrbracket(S)\right) \\
\llbracket \text { if } p=0 \text { then } c_{1} \text { else } c_{2} \rrbracket(S) & =\left\{\sigma \in \llbracket c_{1} \rrbracket(S) \mid \sigma(p)=0\right\} \cup\left\{\sigma \in \llbracket c_{2} \rrbracket(S) \mid \sigma(p) \neq 0\right\} \\
\llbracket \text { while } p \bowtie 0 \text { do } c \rrbracket(S) & =\nu(\lambda X .\{\sigma \in S \mid \sigma(p) \bowtie 0\} \cup\{\sigma \in \llbracket c \rrbracket(X) \mid \sigma(p) \bowtie 0\}),
\end{aligned}
$$

where $\bowtie \in\{=, \neq\}$ and $\nu F$ is the greatest fixed point of $F$. Intuitively, $\sigma \in \llbracket c \rrbracket(S)$ means that executing $c$ from $\sigma$ results in a state in $S$ if the execution terminates; notice that $\sigma$ should be in $\llbracket c \rrbracket(S)$ if $c$ does not terminate. The semantics uses the greatest fixed point instead of the least fixed point in the while statement so that $\llbracket c \rrbracket(S)$ contains the states from which the execution of $c$ does not terminate. If we used the least fixed point in the semantics of a while loop, then only the initial states from which the program terminates would be in the denotation of the loop. For example, consider the following program $P$ that does not terminate for any initial state: while $0=0$ do skip. Then, $\llbracket P \rrbracket(S)$ should be St. However, if the denotation of a while loop were given by the least fixed point, then $\llbracket P \rrbracket(S)$ would be $\emptyset$.

Example 2. Recall the program $c_{\text {fall }}$ in Figure 1. Let $p_{1}$ be $-g t+g t_{0}-v+$ $v_{0}-x \rho+x_{0} \rho, p_{2}$ be $-g t^{2}+g t_{0}^{2}-2 t v+2 t_{0} v_{0}+2 x-2 x_{0}, p$ be $p_{1}+p_{2}$, and $S$ be $\{\sigma \in \mathbf{S t} \mid \sigma(p)=0\}$. We show that $\llbracket c_{\text {fall }} \rrbracket(S)=\mathbf{S t}$. We write $c_{1}$ for $(x$, $v, t):=\left(x_{0}, v_{0}, t_{0}\right)$, and $c_{2}$ for $(x, v, t):=(x+v d t, v-g d t-\rho v d t, t+d t)$. We have $\llbracket c_{\text {fall }} \rrbracket(S)=\llbracket c_{1} \rrbracket\left(\llbracket\right.$ while $t-a \neq 0$ do $\left.c_{2} \rrbracket(S)\right)=\llbracket c_{1} \rrbracket(\nu F)$ where $F(X)=\{\sigma \in S \mid \sigma(t-a)=0\} \cup\left\{\sigma \in \llbracket c_{2} \rrbracket(X) \mid \sigma(t-a) \neq 0\right\}$. It is easy to check that $\llbracket c_{1} \rrbracket(S)=\mathbf{S t}$, so it suffices to show that $\nu F \supseteq S$. This holds because $S$ is a fixed point of $F$. Indeed, $F(S)=\{\sigma \in S \mid \sigma(t-a)=0\} \cup$ $\left\{\sigma \in \llbracket c_{2} \rrbracket(S) \mid \sigma(t-a) \neq 0\right\}=\{\sigma \in S \mid \sigma(t-a)=0\} \cup\{\sigma \in S \mid \sigma(t-a) \neq 0\}=$ $S$ as desired. Note that $\llbracket c_{2} \rrbracket(S)=S$ because $c_{2}$ does not change the value of $p$. 
The abstract semantics is essentially the same as that given by Cachera et al. 4] with a small adjustment. The preorder $\subseteq^{\sharp} \subset \mathcal{P}\left(K\left[x_{1}, \ldots, x_{n}\right]\right) \times \mathcal{P}\left(K\left[x_{1}\right.\right.$, $\left.\left.\ldots, x_{n}\right]\right)$ is defined by $\left.S_{1} \sqsubseteq^{\sharp} S_{2}: \Longleftrightarrow S_{2} \subseteq S_{1}\right]^{7}$. Then $\mathcal{P}\left(K\left[x_{1}, \ldots, x_{n}\right]\right)$ is a complete lattice, and the meet is given as the set unions: Given $H \in \mathcal{P}\left(K\left[x_{1}, \ldots\right.\right.$, $\left.\left.x_{n}\right]\right)$ and $U \subseteq \mathcal{P}\left(K\left[x_{1}, \ldots, x_{n}\right]\right), H \sqsubseteq^{\sharp} G$ for all $G \in U$ if and only if $H \sqsubseteq^{\sharp} \cup U$.

The abstraction $\alpha(S)$ is defined by $\left\{p \in K\left[x_{1}, \ldots, x_{n}\right] \mid \forall \sigma \in S, \sigma(p)=0\right\}$, the polynomials evaluated to 0 under all the states of $S$. The concretization $\gamma(G)$ is defined by $\{\sigma \in \mathbf{S t} \mid \forall p \in G, \sigma(p)=0\}$, the states that evaluate all the polynomials in $G$ to 0 . The pair of $\alpha$ and $\gamma$ constitutes a Galois connection; indeed, both $\alpha(S) \check{\complement}^{\sharp} G$ and $S \subseteq \gamma(G)$ are by definition equivalent to the following: $\forall p \in G, \forall \sigma \in S, \sigma(p)=0$. For example, the set of a state $\left\{\left\{x_{1} \mapsto 1, x_{2} \mapsto 0\right\}\right\}$ is abstracted by the set $\left\{\left(x_{1}-1\right) p_{1}+x_{2} p_{2} \mid p_{1}, p_{2} \in K\left[x_{1}, \ldots, x_{n}\right]\right\}$; this set is equivalently $\left\langle x_{1}-1, x_{2}\right\rangle$, the ideal generated by $x_{1}-1$ and $x_{2}$.

The definition of the abstract semantics is parameterized over a remainderlike operation $\boldsymbol{\operatorname { R e m }}(f, p)$ that satisfies $\boldsymbol{\operatorname { R e m }}(f, p)=f-q p$ for some $q$; we allow any Rem that satisfies this condition to be used. Note that this differs from the standard remainder operation where we require $\operatorname{LM}_{\prec}(p)$ - the greatest monomial in $p$ with respect to a monomial order $\preceq-$ not to divide any monomial in $\mathrm{LM}_{\prec}(\operatorname{Rem}(f, p))$. We write $\operatorname{Rem}(G, p)$, where $G$ is a set of polynomials, for the set $\{\boldsymbol{\operatorname { R e m }}(f, p) \mid f \in G \backslash\{0\}\}$.

The abstract semantics $\llbracket c \rrbracket_{\text {Rem }}^{\sharp}$ is defined as follows.

$$
\begin{aligned}
& \llbracket c \rrbracket_{\text {Rem }}^{\sharp}:\left(\mathcal{P}\left(K\left[x_{1}, \ldots, x_{n}\right]\right), \sqsubseteq^{\sharp}\right) \rightarrow\left(\mathcal{P}\left(K\left[x_{1}, \ldots, x_{n}\right]\right), \sqsubseteq^{\sharp}\right) \\
& \llbracket \text { skip } \rrbracket_{\text {Rem }}^{\sharp}(G)=G \\
& \llbracket x:=p \rrbracket_{\operatorname{Rem}}^{\sharp}(G)=G[x:=p] \\
& \llbracket c_{1} ; c_{2} \rrbracket_{\text {Rem }}^{\sharp}(G)=\llbracket c_{1} \rrbracket_{\text {Rem }}^{\sharp}\left(\llbracket c_{2} \rrbracket_{\text {Rem }}^{\sharp}(G)\right) \\
& \llbracket \text { if } p=0 \text { then } c_{1} \text { else } c_{2} \rrbracket_{\operatorname{Rem}}^{\sharp}(G)=p \cdot \llbracket c_{2} \rrbracket_{\operatorname{Rem}}^{\sharp}(G) \cup \operatorname{Rem}\left(\llbracket c_{1} \rrbracket_{\operatorname{Rem}}^{\sharp}(G), p\right) \\
& \llbracket \text { while } p \neq 0 \text { do } c \rrbracket_{\operatorname{Rem}}^{\sharp}(G)=\nu\left(\lambda H \cdot p \cdot \llbracket c \rrbracket_{\operatorname{Rem}}^{\sharp}(H) \cup \operatorname{Rem}(G, p)\right) \\
& \llbracket \text { while } p=0 \text { do } c \rrbracket_{\text {Rem }}^{\sharp}(G)=\nu\left(\lambda H \cdot p \cdot G \cup \operatorname{Rem}\left(\llbracket c \rrbracket_{\text {Rem }}^{\sharp}(H), p\right)\right) \text {. }
\end{aligned}
$$

In this definition, $G[x:=p]=\{q[x:=p] \mid q \in G\}$ and $q[x:=p]$ is the polynomial obtained by replacing $x$ with $p$ in $q . \nu F$ exists for an arbitrary monotone $F$ because we are working in the complete lattice $\mathcal{P}\left(K\left[x_{1}, \ldots, x_{n}\right]\right)$; concretely, we have $\nu F=\bigcup\left\{G \mid G \sqsubseteq^{\sharp} F(G)\right\}$.

$\llbracket c \rrbracket_{\text {Rem }}^{\sharp}$ transfers backward a set of polynomials whose values are 0. Cachera et al. [4, Theorem 3] showed the soundness of this abstract semantics: For any program $c$ and a set of polynomials $G$, we have $\gamma\left(\llbracket c \rrbracket_{\text {Rem }}^{\sharp}(G)\right) \subseteq \llbracket c \rrbracket(\gamma(G))$. Although our abstract values are sets rather than ideals, we can prove this theorem in the same way (i.e., induction on the structure of $c$ ) as the original proof.

The highlight of the abstract semantics is the definition of $\llbracket$ if $p=0$ then $c_{1}$ else $c_{2} \rrbracket_{\text {Rem }}^{\sharp}$. In order to explain this case, let us describe a part of the sound-

\footnotetext{
7 The original abstract semantics of Cachera et al. 4 is defined as a transformer on ideals of polynomials; however, we formulate it here so that it operates on sets of polynomials because their invariant-synthesis algorithm depends on the choice of a generator of an ideal.
} 
ness proof: We show $\gamma\left(\llbracket\right.$ if $p=0$ then $c_{1}$ else $\left.c_{2} \rrbracket_{\operatorname{Rem}}^{\sharp}(G)\right) \subseteq \llbracket$ if $p=0$ then $c_{1}$ else $c_{2} \rrbracket(\gamma(G))$ assuming $\gamma\left(\llbracket c_{1} \rrbracket_{\text {Rem }}^{\sharp}(G)\right) \subseteq \llbracket c_{1} \rrbracket(\gamma(G))$ and $\gamma\left(\llbracket c_{2} \rrbracket_{\text {Rem }}^{\sharp}(G)\right) \subseteq$ $\llbracket c_{2} \rrbracket(\gamma(G))$. Suppose $\sigma \in \gamma\left(\llbracket\right.$ if $p=0$ then $c_{1}$ else $\left.c_{2} \rrbracket_{\text {Rem }}^{\sharp}(G)\right)$. Our goal is to show $\sigma \in \llbracket$ if $p=0$ then $c_{1}$ else $c_{2} \rrbracket(\gamma(G))$. Therefore, it suffices to show that (1) $\sigma(p)=0$ implies $\sigma \in \llbracket c_{1} \rrbracket(\gamma(G))$, and $(2) \sigma(p) \neq 0$ implies $\sigma \in \llbracket c_{2} \rrbracket(\gamma(G))$.

- We first show that if $\sigma(p)=0$ then $\sigma \in \llbracket c_{1} \rrbracket(\gamma(G))$. By the induction hypothesis, we have $\gamma\left(\llbracket c_{1} \rrbracket_{\text {Rem }}^{\sharp}(G)\right) \subseteq \llbracket c_{1} \rrbracket(\gamma(G))$, so it suffices to show that $\sigma \in \gamma\left(\llbracket c_{1} \rrbracket_{\text {Rem }}^{\sharp}(G)\right)$. Take $f \in \llbracket c_{1} \rrbracket_{\text {Rem }}^{\sharp}(G)$. Then there exists $r \in$ $\operatorname{Rem}\left(\llbracket c_{1} \rrbracket_{\mathbf{R e m}}^{\sharp}(G), p\right)$ and $q \in K\left[x_{1}, \ldots, x_{n}\right]$ such that $f=q p+r$. Because $\sigma(p)=0$ and $r \in \operatorname{Rem}\left(\llbracket c_{1} \rrbracket_{\mathbf{R e m}}^{\sharp}(G), p\right) \subseteq \llbracket$ if $p=0$ then $c_{1}$ else $c_{2} \rrbracket_{\mathbf{R e m}}^{\sharp}(G)$ and $\sigma \in \llbracket$ if $p=0$ then $c_{1}$ else $c_{2} \rrbracket_{\operatorname{Rem}}^{\sharp}(G)$, we have $\sigma(f)=\sigma(q) \sigma(p)+\sigma(r)=$ 0 . Since $f$ is an arbitrary element of $\llbracket c_{1} \rrbracket_{\text {Rem }}^{\sharp}(G)$, by definition of $\gamma$ we conclude that $\sigma \in \gamma\left(\llbracket c_{1} \rrbracket_{\text {Rem }}^{\sharp}(G)\right)$.

- Next we show that $\sigma(p) \neq 0$ implies $\sigma \in \llbracket c_{2} \rrbracket(\gamma(G))$. By the induction hypothesis, we have $\gamma\left(\llbracket c_{2} \rrbracket_{\text {Rem }}^{\sharp}(G)\right) \subseteq \llbracket c_{2} \rrbracket(\gamma(G))$, so it suffices to show that $\sigma \in \gamma\left(\llbracket c_{2} \rrbracket_{\text {Rem }}^{\sharp}(G)\right)$. Take $f \in \llbracket c_{2} \rrbracket_{\text {Rem }}^{\sharp}(G)$. Then $p f \in p \cdot \llbracket c_{2} \rrbracket_{\text {Rem }}^{\sharp}(G) \subseteq$ $\llbracket$ if $p=0$ then $c_{1}$ else $c_{2} \rrbracket_{\text {Rem }}^{\sharp}(G)$, thus $\sigma(p f)=0$. From the assumption $\sigma(p) \neq 0$, this implies $\sigma(f)=0$. Since $f$ is arbitrary, we conclude that $\sigma \in \gamma\left(\llbracket c_{2} \rrbracket_{\text {Rem }}^{\sharp}(G)\right)$ 8.

The abstract semantics is related to the postcondition problem as follows:

Theorem 1. If $\llbracket c \rrbracket_{\mathbf{R e m}}^{\sharp}(G)=\{0\}$, then $\llbracket c \rrbracket(\gamma(G))=$ St (hence $g=0$ is a solution of the postcondition problem for any $g \in G)$.

Proof. From the soundness above, $\gamma\left(\llbracket c \rrbracket_{\text {Rem }}^{\sharp}(G)\right)=\gamma(\{0\})=\mathbf{S t} \subseteq \llbracket c \rrbracket(\gamma(G))$; therefore $\llbracket c \rrbracket(\gamma(G))=\mathbf{S t}$ follows because $\mathbf{S t}$ is the top element in the concrete domain.

Example 3. We exemplify how the abstract semantics works using the program $c_{f a l l}$ in Figure 1. Set $p, c_{1}$, and $c_{2}$ as in Example 2. Define Rem in this example by $\boldsymbol{\operatorname { R e m }}(f, p)=f$. First, let $F(H):=(t-a) \llbracket c_{2} \rrbracket_{\mathbf{R e m}}^{\sharp}(H) \cup\{p\}$, $g_{0}:=1, g_{n+1}:=(t-a)(t+d t-a) \ldots(t+n d t-a)$, and $G=\left\{g_{n} p \mid n \in \mathbb{N}\right\}$. Then $\nu F=G$. Indeed, by definition of $\sqsubseteq^{\sharp}$, we have $\top=\emptyset$, and it is easy to check that $F^{n}(\top)=\left\{g_{k} p \mid 0 \leq k<n\right\}$. Therefore we have $\nu F \sqsubseteq^{\sharp} G$, because $G$ is the greatest lower bound of $\left(F^{n}(\top)\right)_{n \in \mathbb{N}}$. By simple computation, we can see that $G$ is a fixed point of $F$, so we also have $G \sqsubseteq^{\sharp} \nu F$; hence, $\nu F=$ $G$. Therefore, $\llbracket c_{\text {fall }} \rrbracket_{\text {Rem }}^{\sharp}(\{p\})=\{0\}: \llbracket c_{\text {fall }} \rrbracket_{\text {Rem }}^{\sharp}(\{p\})=\llbracket c_{1} \rrbracket_{\text {Rem }}^{\sharp}(\llbracket$ while $t-$ $a \neq 0$ do $\left.c_{2} \rrbracket_{\text {Rem }}^{\sharp}(\{p\})\right)=\llbracket c_{1} \rrbracket_{\text {Rem }}^{\sharp}\left(\nu\left(\lambda H .(t-a) \llbracket c_{2} \rrbracket_{\text {Rem }}^{\sharp}(H) \cup \operatorname{Rem}(\{p\}, t-\right.\right.$ $a)))=\llbracket c_{1} \rrbracket_{\text {Rem }}^{\sharp}\left(\nu\left(\lambda H .(t-a) \llbracket c_{2} \rrbracket_{\text {Rem }}^{\sharp}(H) \cup\{p\}\right)\right)=\llbracket c_{1} \rrbracket_{\text {Rem }}^{\sharp}\left(\left\{g_{n} p \mid n \in \mathbb{N}\right\}\right)=$ $\left\{\left(g_{n} p\right)\left[x:=x_{0}, v:=v_{0}, t:=t_{0}\right] \mid n \in \mathbb{N}\right\}=\{0\}$.

${ }^{8}$ The soundness would still hold even if we defined $\llbracket$ if $p=0$ then $c_{1}$ else $c_{2} \rrbracket_{\operatorname{Rem}}^{\sharp}(G)$ by $\llbracket c_{2} \rrbracket_{\text {Rem }}^{\sharp}(G) \cup \operatorname{Rem}\left(\llbracket c_{1} \rrbracket_{\text {Rem }}^{\sharp}(G), p\right)$ instead of $p \cdot \llbracket c_{2} \rrbracket_{\operatorname{Rem}}^{\sharp}(G) \cup \operatorname{Rem}\left(\llbracket c_{1} \rrbracket_{\text {Rem }}^{\sharp}(G), p\right)$. The multiplier $p$ makes the abstract semantics more precise. 
By Theorem 1, a set of polynomials $G$ such that $\llbracket c \rrbracket_{\text {Rem }}^{\sharp}(G)=\{0\}$ for some Rem constitutes a solution of the postcondition problem. The choice of Rem indeed matters in solving the postcondition problem: There are $c$ and $G$ such that $\llbracket c \rrbracket_{\text {Rem }}^{\sharp}(G)=\{0\}$ holds for some Rem but not for others. The reader is referred to [4, Section 4.1] for a concrete example.

\section{Generalized homogeneous polynomials}

\subsection{Definition}

A polynomial $p$ is said to be a homogeneous polynomial of degree $d$ if the degree of every monomial in $p$ is $d$ [5. As we mentioned in Section 1, we generalize this notion of homogeneity.

We first generalize the notion of the degree of a polynomial.

Definition 1. The group of generalized degrees (g-degrees) $\mathbf{G D e g}_{B}$, ranged over by $\tau$, is an Abelian group freely generated by the finite set $B$; that is, $\operatorname{GDeg}_{B}:=\left\{b_{1}^{n_{1}} \ldots b_{m}^{n_{m}} \mid b_{1}, \ldots, b_{m} \in B, n_{1}, \ldots, n_{m} \in \mathbb{Z}\right\}$. We call $B$ the set of the base degrees. We often omit $B$ in $\mathbf{G D e g}_{B}$ if the set of the base degrees is clear from the context.

For example, if we set $B$ to $\{L, T\}$, then $L, T$, and $L T^{-1}$ are all generalized degrees. By definition, $\operatorname{GDeg}_{B}$ has the multiplication on these g-degrees (e.g., $(L T) \cdot\left(L T^{-2}\right)=L^{2} T^{-1}$ and $\left.\left(L T^{2}\right)^{2}=L^{2} T^{4}\right)$.

In the analogy of quantity dimen-

$$
\begin{gathered}
\Gamma \vdash \text { skip } \\
\frac{\Gamma \vdash c_{1} \quad \Gamma \vdash c_{2}}{\Gamma \vdash c_{1} ; c_{2}} \\
\frac{\Gamma(x)=\operatorname{gdeg}_{\Gamma}(p)}{\Gamma \vdash x:=p}
\end{gathered}
$$
sions, the set $B$ corresponds to the base quantity dimensions (e.g., $L$ for lengths and $T$ for times); the set GDeg $_{B}$ corresponds to the derived quantity dimensions (e.g., $L T^{-1}$ for velocities and $L T^{-2}$ for acceleration rates.); multiplication expresses the

(T-Assign) relationship among quantity dimen-

$\frac{\operatorname{gdeg}_{\Gamma}(p)=\tau \quad \Gamma \vdash c_{1} \quad \Gamma \vdash c_{2}}{\Gamma \vdash \text { if } p=0 \text { then } c_{1} \text { else } c_{2}}$

$$
\frac{\operatorname{gdeg}_{\Gamma}(p)=\tau \quad \Gamma \vdash c}{\Gamma \vdash \text { while } p \bowtie 0 \text { do } c}
$$

(T-While)

Fig. 2. Typing rules $\operatorname{gdeg}_{\Gamma}(k w)$, the $g$-degree of a monomial $k w$ under $\Gamma$, is defined by $\operatorname{gdeg}_{\Gamma}(w)$. 
For example, set $\Gamma$ to $\left\{t \mapsto T, v \mapsto L T^{-1}\right\}$; then $\operatorname{gdeg}_{\Gamma}(2 v t)=L$. In terms of the analogy with quantity dimensions, this means that the expression $2 v t$ represents a length.

Definition 3. We say $p$ is a generalized homogeneous (GH) polynomial of gdegree $\tau$ under $\Gamma$ if every monomial in $p$ has the g-degree $\tau$ under $\Gamma$. We write $\operatorname{gdeg}_{\Gamma}(p)$ for the $g$-degree of $p$ if it is a GH polynomial under $\Gamma$; if it is not, then $\operatorname{gdeg}_{\Gamma}(p)$ is not defined. We write $K\left[x_{1}, \ldots, x_{n}\right]_{\Gamma, \tau}$ for the set of the GH polynomials with $g$-degree $\tau$ under $\Gamma$. We write $K\left[x_{1}, \ldots, x_{n}\right]_{\Gamma}$ for $\bigcup_{\tau \in \mathbf{G D e g}} K\left[x_{1}\right.$, $\left.\ldots, x_{n}\right]_{\Gamma, \tau}$.

Example 4. The polynomial $-g t^{2}+g t_{0}^{2}-2 t v+2 t_{0} v_{0}+2 x-2 x_{0}$ (the polynomial $p_{2}$ in Example 2) is a GH-polynomial under

$\Gamma:=\left\{g \mapsto L T^{-2}, t \mapsto T, v \mapsto L T^{-1}, x \mapsto L, x_{0} \mapsto L, v_{0} \mapsto L T^{-1}, \rho \mapsto T^{-1}, a \mapsto T\right\}$

because all the monomials in $p_{2}$ have the same g-degree in common; for example, $\operatorname{gdeg}_{\Gamma}\left(-g t^{2}\right)=\Gamma(g) \Gamma(t)^{2}=\left(L T^{-2}\right) T^{2}=L ; \operatorname{gdeg}_{\Gamma}(-2 t v)=\Gamma(t) \Gamma(v)=$ $T\left(L T^{-1}\right)=L ; \operatorname{gdeg}_{\Gamma}(2 x)=\Gamma(x)=L ;$ and $\operatorname{gdeg}_{\Gamma}\left(-2 x_{0}\right)=\Gamma\left(x_{0}\right)=L$. Therefore, $\operatorname{gdeg}_{\Gamma}\left(p_{2}\right)=L$. We also have $\operatorname{gdeg}_{\Gamma}\left(p_{1}\right)=L T^{-1}$.

It is easy to see that any $p \in K\left[x_{1}, \ldots, x_{n}\right]$ can be uniquely written as the finite sum of GH polynomials as $p_{\Gamma, \tau_{1}}+\cdots+p_{\Gamma, \tau_{m}}$, where $p_{\Gamma, \tau_{i}}$ is the summand of g-degree $\tau_{i}$ under $\Gamma$ in this representation. For example, the polynomial $p$ in Example 2, can be written as $p_{L}+p_{L T^{-1}}$ where $p_{L}=p_{1}$ and $p_{L T^{-1}}=p_{2}$ from the previous example. We call $p_{\Gamma, \tau}$ the homogeneous component of $p$ with $g$-degree $\tau$ under $\Gamma$, or simply a homogeneous component of $p$; we often omit $\Gamma$ part if it is clear from the context.

The definitions above are parameterized over a g-degree assignment $\Gamma$. It is determined from the usage of variables in a given program, which is captured by the following type judgment.

Definition 4. The judgment $\Gamma \vdash c$ is the smallest relation that satisfies the rules in Figure Q We say $\Gamma$ is consistent with the program $c$ if $\Gamma \vdash c$ holds.

The consistency relation above is an adaptation of the dimension type system proposed by Kennedy [12,13, to our imperative language. A g-degree assignment $\Gamma$ such that $\Gamma \vdash c$ holds makes every polynomial in $c$ a GH one. In the rule T-Assign, we require the polynomial $p$ to have the same g-degree as that of $x$ in $\Gamma$.

\subsection{Automated inference of the g-degree assignment}

Kennedy also proposed a constraint-based automated type inference algorithm of his type system [12,13. We adapt his algorithm so that, given a command $c$, it infers a g-degree assignment $\Gamma$ such that $\Gamma \vdash c$. The algorithm is in three steps: (1) designating a template of the g-degree assignment, (2) generating constraints over g-degrees, and (3) solving the constraints. In order to make the current paper self-contained, we explain each step below. 
Step 1: Designating a template of the $g$-degree assignment Let $S_{c}:=\left\{x_{1}, \ldots, x_{n}\right\}$ be the set of the variables occurring in the given program $c$. Then, the algorithm first designates a template g-degree assignment $\Gamma_{c}:=\left\{x_{1} \mapsto \alpha_{x_{1}}, \ldots, x_{n} \mapsto \alpha_{x_{n}}\right\}$ where $\alpha_{x_{1}}, \ldots, \alpha_{x_{n}}$ are fresh unknowns taken from the set $\mathbf{G D e g} \mathbf{V}$ for the gdegrees of $x_{1}, \ldots, x_{n}$. For example, given the program $c_{\text {fall }}$ in Figure 1, the algorithm designates

$$
\Gamma_{c_{\text {fall }}}:=\left\{\begin{array}{l}
g \mapsto \alpha_{g}, t \mapsto \alpha_{t}, d t \mapsto \alpha_{d t}, v \mapsto \alpha_{v}, x \mapsto \alpha_{x}, \\
x_{0} \mapsto \alpha_{x_{0}}, v_{0} \mapsto \alpha_{v_{0}}, \rho \mapsto \alpha_{\rho}, a \mapsto \alpha_{a}
\end{array}\right\}
$$

where $\alpha_{g}, \alpha_{t}, \alpha_{d t}, \alpha_{v}, \alpha_{x}, \alpha_{x_{0}}, \alpha_{v_{0}}, \alpha_{\rho}, \alpha_{a}$ are distinct unknowns for the g-degrees of the variables that are to be inferred.

Step 2: Generating constraints over g-degrees The algorithm then generates the constraints over the g-degrees. We first define the set of constraints. Let GDeg' be $\operatorname{GDeg}_{\left\{\alpha_{1}, \ldots, \alpha_{n}\right\}}$ in the rest of this section, where $\alpha_{1}, \ldots, \alpha_{n}$ are the unknowns generated in the previous step. (Recall that $\mathbf{G D e g}_{S}$ is the set of g-degrees generated by $S$. Therefore, GDeg' is the set of products of the form $\alpha_{1}^{k_{1}} \ldots \alpha_{n}^{k_{n}}$ for $k_{1}, \ldots, k_{n} \in \mathbb{Z}$.) The $\Gamma_{c}$ generated in the previous step can be seen as a map from Var to GDeg'.

A $g$-degree constraint is an equation $\tau_{1}=\tau_{2}$ where $\tau_{1}, \tau_{2} \in \mathbf{G D e g}^{\prime}$. We use a metavariable $\sigma$ for maps from $\left\{\alpha_{1}, \ldots, \alpha_{n}\right\}$ to $\mathbf{G D e g}_{B}$. This map can be naturally extended to take the elements of $\mathbf{G D e g}^{\prime}$. We say that $\sigma$ is a solution of a constraint set $C$ if it satisfies all the equations in $C$. For example, the map $\sigma:=\left\{\alpha_{v} \mapsto L T^{-1}, \alpha_{x} \mapsto L, \alpha_{t} \mapsto T\right\}$ is a solution of the constraint set $\left\{\alpha_{v}=\alpha_{x} \alpha_{t}^{-1}\right\}$ since $\sigma\left(\alpha_{v}\right)=L T^{-1}=\sigma\left(\alpha_{x} \alpha_{t}^{-1}\right)=\sigma\left(\alpha_{x}\right) \sigma\left(\alpha_{t}\right)^{-1}$.

For a polynomial $p:=a_{1} w_{1}+\cdots+a_{n} w_{n}$, we write $\operatorname{gdeg}_{\Gamma}^{\prime}(p)$ for the pair $\left.\operatorname{gdeg}_{\Gamma}\left(w_{1}\right), C\right)$ where $C$ is $\emptyset$ if $n=1$ and $\left\{\operatorname{gdeg}_{\Gamma}\left(w_{1}\right)=\operatorname{gdeg}_{\Gamma}\left(w_{2}\right), \ldots\right.$, $\left.\operatorname{gdeg}_{\Gamma}\left(w_{n-1}\right)=\operatorname{gdeg}_{\Gamma}\left(w_{n}\right)\right\}$ otherwise. The intuition of $\operatorname{gdeg}_{\Gamma}^{\prime}(p)=(\tau, C)$ is that, for any solution $\sigma$ of $C$, the polynomial $p$ is generalized homogeneous and its g-degree is $\sigma(\tau)$.

For example, let $\Gamma$ be $\left\{v \mapsto \alpha_{v}, g \mapsto \alpha_{g}, x \mapsto \alpha_{x}\right\}$ and $p$ be $2 v^{2}+g x$; then, $\operatorname{gdeg}_{\Gamma}^{\prime}(p)$ is the pair $\left(\alpha_{v}^{2}, C\right)$ where $C$ is $\left\{\alpha_{v}^{2}=\alpha_{g} \alpha_{x}\right\}$. For a solution $\sigma:=$ $\left\{\alpha_{v} \mapsto L T^{-1}, \alpha_{g} \mapsto L T^{-2}, \alpha_{x} \mapsto L\right\}$ of $C, \sigma(\Gamma)=\left\{v \mapsto L T^{-1}, g \mapsto L T^{-2}, x \mapsto\right.$ $L\}$. The polynomial $p$ is generalized homogeneous under $\sigma(\Gamma)$ since $\sigma(\Gamma)\left(v^{2}\right)=$ $\sigma(\Gamma)(g x)=L^{2} T^{-2}$. This is equal to $\sigma\left(\alpha_{v}^{2}\right)$.

The function $P T$ for the constraint generation is defined as follows:

$$
\begin{aligned}
& P T(\Gamma, \text { skip }):=\emptyset \\
& P T\left(\Gamma, c_{1} ; c_{2}\right):=P T\left(\Gamma, c_{1}\right) \cup P T\left(\Gamma, c_{2}\right) \\
& P T(\Gamma, x:=p):=\{\Gamma(x)=\tau\} \cup C \\
& \text { where }(\tau, C):=\operatorname{gdeg}_{\Gamma}^{\prime}(p) \\
& P T\left(\Gamma, \text { if } p=0 \text { then } c_{1} \text { else } c_{2}\right):=C \cup P T\left(\Gamma, c_{1}\right) \cup P T\left(\Gamma, c_{2}\right) \text { where }(\tau, C):=\operatorname{gdeg}_{\Gamma}^{\prime}(p) \\
& P T(\Gamma, \text { while } p \bowtie 0 \text { do } c):= P T(\Gamma, c) \\
& \text { where }(\tau, C):=\operatorname{gdeg}_{\Gamma}^{\prime}(p) .
\end{aligned}
$$


The constraints $P T(\Gamma, c)$ is defined so that its any solution $\sigma$ satisfies $\sigma(\Gamma) \vdash$ $c$. The definition essentially constructs the derivation tree of $\Gamma \vdash c$ following the rules in Figure 2 and collects the constraints appearing in the tree.

Example 5. PT $\left(\Gamma_{c_{\text {fall }}}, c_{\text {fall }}\right)$ generates the following constraints. From the commands in Line 1, the constraint set $\left\{\alpha_{x}=\alpha_{x_{0}}, \alpha_{v}=\alpha_{v_{0}}, \alpha_{t}=\alpha_{t_{0}}\right\}$ is generated; from the guard in Line 2, $\left\{\alpha_{t}=\alpha_{a}\right\}$ is generated; from the right-hand side of Line 3 , the constraint set $\left\{\alpha_{x}=\alpha_{v} \alpha_{d t}, \alpha_{v}=\alpha_{g} \alpha_{d t}, \alpha_{g} \alpha_{d t}=\alpha_{\rho} \alpha_{v} \alpha_{d t}, \alpha_{t}=\alpha_{d t}\right\}$, which ensures the generalized homogeneity of each polynomial, is generated; PT also generates $\left\{\alpha_{x}=\alpha_{x}, \alpha_{v}=\alpha_{v}, \alpha_{t}=\alpha_{t}\right\}$, which ensures that the g-degrees of the left-hand side and the right-hand side are identical.

Step 3: Solving the constraints The algorithm then calculates a solution of the generated constraints. The constraint-solving procedure is almost the same as that by Kennedy [12, Section 5.2], which is based on Lankford's unification algorithn 14 .

The procedure obtains a solution $\sigma$ from the given constraint set $C$ by applying the following rewriting rules successively:

$$
\begin{aligned}
\left(\left\{\alpha^{\prime k} \boldsymbol{\alpha}^{n}=1\right\} \cup C, \sigma\right) \rightarrow & \left(\left\{\alpha^{\prime} \mapsto \boldsymbol{\alpha}^{-\frac{n}{k}}\right\}(C),\left\{\alpha^{\prime} \mapsto \boldsymbol{\alpha}^{-\frac{n}{k}}\right\} \circ \sigma\right) \\
& \text { where the absolute value of } k \text { is not more than those of } \boldsymbol{n} \\
& (\text { if } k \text { divides all the integers in } \boldsymbol{n}) \\
\left(\left\{\alpha^{\prime k} \boldsymbol{\alpha}^{\boldsymbol{n}}=1\right\} \cup C, \sigma\right) \rightarrow & \left(\left\{\omega^{k} \boldsymbol{\alpha}^{\boldsymbol{n} \text { mod } k}=1\right\} \cup \sigma^{\prime}(C), \sigma^{\prime} \circ \sigma\right) \\
& \text { where the absolute value of } k \text { is not more than those of } \boldsymbol{n}, \\
& \sigma^{\prime}=\left\{\alpha^{\prime} \mapsto \omega \boldsymbol{\alpha}^{-\left\lfloor\frac{n}{k}\right\rfloor}\right\}, \\
& \text { and } \omega \text { is a fresh element of } \mathbf{G D e g V} \\
& \text { (if there is an integer in } \boldsymbol{n} \text { that is not divisible by } k) \\
(\{1=1\} \cup C, \sigma) \rightarrow & (C, \sigma) \\
\left(\left\{\tau_{1}=\tau_{2}\right\} \cup C, \sigma\right) \rightarrow & \left(\left\{\tau_{1} \tau_{2}^{-1}=1\right\} \cup C, \sigma\right) \\
C \rightarrow & (C, \emptyset) .
\end{aligned}
$$

The idea of the procedure is to construct a solution iteratively converting a constraint $\alpha^{\prime k} \boldsymbol{\alpha}^{n}=1$ to $\left\{\alpha^{\prime} \mapsto \boldsymbol{\alpha}^{-\frac{n}{k}}\right\}$ if $k$ divides all the integers in $\boldsymbol{n}$ (i.e., the second case). If $k$ does not (i.e., the third case 10 , the procedure (1) splits $\frac{n}{k}$ to the quotient $\left\lfloor\frac{n}{k}\right\rfloor$ and the remainder $\boldsymbol{n} \bmod k,(2)$ generates a fresh g-degree variable $\omega$ representing $\boldsymbol{\alpha}^{-\frac{n \bmod k}{k}}$, and (3) sets $\alpha^{\prime}$ in the solution to $\omega \boldsymbol{\alpha}^{-\left\lfloor\frac{n}{k}\right\rfloor}$ which is equal to $\boldsymbol{\alpha}^{-\frac{n}{k}}$.

After obtaining a solution with the procedure above, the inference algorithm assigns different base degree to each surviving g-degree variable.

Example 6. Consider the following constraint set $C$ :

$$
\left\{\begin{array}{l}
\alpha_{x} \alpha_{x_{0}}^{-1}=1, \alpha_{v} \alpha_{v_{0}}^{-1}=1, \alpha_{t} \alpha_{t_{0}}^{-1}=1, \alpha_{t} \alpha_{d t}^{-1}=1 \\
\alpha_{x} \alpha_{v}^{-1} \alpha_{d t}^{-1}=1, \alpha_{v} \alpha_{g}^{-1} \alpha_{d t}^{-1}=1, \alpha_{g} \alpha_{d t} \alpha_{\rho}^{-1} \alpha_{v}^{-1} \alpha_{d t}^{-1}=1
\end{array}\right\}
$$

\footnotetext{
${ }^{9}$ We do not discuss the termination of the procedure in this paper. See Kennedy [12, Section 5.2].

${ }^{10}$ We do not use this case in the rest of this paper.
} 
which is equivalent to that of Example 5. After several steps of rewriting, the procedure obtains

$$
\left(\left\{\begin{array}{l}
\alpha_{v_{0}} \alpha_{g}^{-1} \alpha_{d t}^{-1}=1, \\
\alpha_{g} \alpha_{\rho}^{-1} \alpha_{v_{0}}^{-1}=1
\end{array}\right\},\left\{\begin{array}{l}
\alpha_{x} \mapsto \alpha_{v_{0}} \alpha_{d t}, \alpha_{v} \mapsto \alpha_{v_{0}}, \\
\alpha_{t} \mapsto \alpha_{d t}, \alpha_{t_{0}} \mapsto \alpha_{d t}, \\
\alpha_{x_{0}} \mapsto \alpha_{v_{0}} \alpha_{d t}
\end{array}\right\}\right) .
$$

At the next step, suppose that the procedure picks up the constraint $\alpha_{v_{0}} \alpha_{g}^{-1} \alpha_{d t}^{-1}=$ 1. By applying the second rule, the procedure generates the following state

$$
\left(\left\{\alpha_{\rho}^{-1} \alpha_{d t}^{-1}=1\right\},\left\{\begin{array}{l}
\alpha_{x} \mapsto \alpha_{g} \alpha_{d t}^{2}, \alpha_{v} \mapsto \alpha_{g} \alpha_{d t}, \\
\alpha_{t} \mapsto \alpha_{d t}, \alpha_{t_{0}} \mapsto \alpha_{d t}, \\
\alpha_{x_{0}} \mapsto \alpha_{g} \alpha_{d t}^{2}, \alpha_{v_{0}} \mapsto \alpha_{g} \alpha_{d t}
\end{array}\right\}\right) .
$$

Then, with the second and last rules, the procedure obtains the following solution:

$$
\left\{\begin{array}{l}
\alpha_{x} \mapsto \alpha_{g} \alpha_{d t}^{2}, \alpha_{v} \mapsto \alpha_{g} \alpha_{d t}, \\
\alpha_{t} \mapsto \alpha_{d t}, \alpha_{t_{0}} \mapsto \alpha_{d t} \\
\alpha_{x_{0}} \mapsto \alpha_{g} \alpha_{d t}^{2}, \alpha_{v_{0}} \mapsto \alpha_{g} \alpha_{d t}, \alpha_{\rho} \mapsto \alpha_{d t}^{-1}
\end{array}\right\}
$$

By assigning the base degree $A$ to $\alpha_{g}$ and $T$ to $\alpha_{d t}$, we have the following solution:

$$
\left\{\begin{array}{l}
\alpha_{x} \mapsto A T^{2}, \alpha_{v} \mapsto A T, \alpha_{t} \mapsto T, \alpha_{t_{0}} \mapsto T, \\
\alpha_{x_{0}} \mapsto A T^{2}, \alpha_{v_{0}} \mapsto A T, \alpha_{\rho} \mapsto T^{-1}
\end{array}\right\}
$$

Notice the set of base degrees is different from that we used in Example 4. in this example, the g-degree for the acceleration rates $(A)$ is used as a base degree, whereas that for lengths $(L)$ is used in Example 4. This happens because the order of the constraints chosen in an execution of the inference algorithm is nondeterministic. Our results in the rest of this paper do not depend on a specific choice of base degrees.

Limitation A limitation of the current g-degree inference algorithm is that, even if a constant symbol in a program is intended to be of a g-degree other than 1 , it has to be of g-degree 1 in the current type system. For example, consider the program $c_{\text {fall }}^{\prime}$ obtained by replacing $g$ in $c_{\text {fall }}$ with 9.81 and $\rho$ with 0.24 . Then, the g-degrees of $v$ and $d t$ are inferred to be 1 due to the assignment $v:=$ $v-9.8 d t-0.24 v d t$ in $c_{f a l l}^{\prime}$ : The constraints for this assignment generated by the inference algorithm is $\left\{\alpha_{v}=\alpha_{d t}, \alpha_{d t}=\alpha_{v} \alpha_{d t}, \alpha_{v}=\alpha_{v}\right\}$, whose only solution is $\left\{\alpha_{v} \mapsto 1, \alpha_{d t} \mapsto 1\right\}$. This degenerated g-degrees are propagated to the other variables during the inference of $c_{\text {fall }}^{\prime}$, leading to the g-degree assignment in which all the variables have the g-degree 1 . This g-degree assignment is not useful for the template-size reduction; any polynomial is a GH polynomial under this assignment.

As a workaround, our current implementation that will be described in Section 7 uses an extension that can assign a g-degree other than 1 to each occurrence of a constant symbol by treating a constant symbol as a variable. For example, for the following program sumpower ${ }_{d}:(x, y, s):=(X+1,0, \underline{1}) ;$ while $x \neq$ 
0 do if $y=0 \operatorname{then}(x, y):=(x-1, x) \operatorname{else}(s, y):=\left(s+y^{d}, y-1\right)$, the inference algorithm treats the underlined occurrence of 1 as a variable and assigns $T^{d}$ to it; the other occurrences of 0 and 1 are given g-degree $T$. This g-degree assignment indeed produces a smaller template.

\section{Abstract semantics restricted to $\mathrm{GH}$ polynomials}

This section gives the main result of this paper: If there is an algebraic invariant of $c$ and $\Gamma \vdash c$, then there exists an algebraic invariant that consists of a GH polynomial under $\Gamma$.

To state this result formally, we revise our abstract semantics by restricting it to the domain of the GH polynomials. The domain is obtained by replacing the underlying set of the domain $\mathcal{P}\left(K\left[x_{1}, \ldots, x_{n}\right]\right)$ with $\mathcal{P}\left(K\left[x_{1}, \ldots, x_{n}\right]_{\Gamma}\right)$. This is a subset of $\mathcal{P}\left(K\left[x_{1}, \ldots, x_{n}\right]\right)$ that is closed under arbitrary meets. We can define the abstraction and the concretization in the same way as in Section 3 .

The revised abstract semantics $\llbracket c \rrbracket_{\text {Rem, }}^{\sharp H}$, which we hereafter call $G H a b$ stract semantics, is the same as the original one except that it is parameterized over the g-degree assignment $\Gamma$. In the following definition, we write $\operatorname{Rem}(G, p)$ for $\left\{\boldsymbol{\operatorname { R e m }}(f, p) \mid f \in\left(G \cap K\left[x_{1}, \ldots, x_{n}\right]_{\Gamma}\right) \backslash\{0\}\right\}$, the set of the remainder obtained from a GH polynomial in $G$ and $p$. We assume that our choice of Rem is a remainder operation such that whenever both $f$ and $p$ are GH polynomials, so is $\boldsymbol{\operatorname { R e m }}(f, p)$.

$$
\begin{aligned}
& \llbracket \text { skip } \rrbracket_{\text {Rem }, \Gamma}^{\sharp \mathrm{H}}(G)=G \\
& \llbracket x:=p \rrbracket_{\operatorname{Rem}, \Gamma}^{\sharp \mathrm{H}}(G)=G[x:=p] \\
& \llbracket c_{1} ; c_{2} \rrbracket_{\mathbf{R e m}, \Gamma}^{\sharp \mathrm{H}}(G)=\llbracket c_{1} \rrbracket_{\mathbf{R e m}, \Gamma}^{\sharp \mathrm{H}}\left(\llbracket c_{2} \rrbracket_{\mathbf{R e m}, \Gamma}^{\sharp \mathrm{H}}(G)\right) \\
& \llbracket \text { if } p=0 \text { then } c_{1} \text { else } c_{2} \rrbracket_{\operatorname{Rem}, \Gamma}^{\sharp \mathrm{H}}(G)=p \cdot \llbracket c_{2} \rrbracket_{\operatorname{Rem}, \Gamma}^{\sharp \mathrm{H}}(G) \cup \operatorname{Rem}\left(\llbracket c_{1} \rrbracket_{\operatorname{Rem}, \Gamma}^{\sharp \mathrm{H}}(G), p\right) \\
& \llbracket \text { while } p \neq 0 \text { do } c \rrbracket_{\operatorname{Rem}, \Gamma}^{\sharp \mathrm{H}}(G)=\nu\left(\lambda H \cdot p \cdot \llbracket c \rrbracket_{\operatorname{Rem}, \Gamma}^{\sharp \mathrm{H}}(H) \cup \operatorname{Rem}(G, p)\right) \\
& \llbracket \text { while } p=0 \text { do } c \rrbracket_{\operatorname{Rem}, \Gamma}^{\sharp H}(G)=\nu\left(\lambda H \cdot p \cdot G \cup \operatorname{Rem}\left(\llbracket c \rrbracket_{\mathbf{R e m}, \Gamma}^{\sharp H}(H), p\right)\right) \text {. }
\end{aligned}
$$

The following theorem guarantees that the invariant found using the semantics $\llbracket c \rrbracket_{\text {Rem }, \Gamma}^{\sharp H}$ is indeed an invariant of $c$.

Theorem 2 (Soundness of the GH abstract semantics).

If $\Gamma \vdash c$ and $G$ is a set of $G H$ polynomials under $\Gamma$, then $\llbracket c \rrbracket_{\mathbf{R e m}, \Gamma}^{\sharp \mathrm{H}}(G)=$ $\llbracket c \rrbracket_{\text {Rem }}^{\sharp}(G)$.

Proof. By induction on $c$.

This theorem implies that if $g$ is a GH polynomial under $\Gamma$ and $\llbracket c \rrbracket_{\operatorname{Rem}, \Gamma}^{\sharp H}(g)=$ $\{0\}$, then $g$ is indeed a solution of the postcondition problem.

Completeness of $\llbracket c \rrbracket_{\text {Rem, } \Gamma}^{\sharp \mathrm{H}}$ is obtained as a corollary of the following lemma.

Lemma 1. Suppose $\Gamma \vdash c, g_{1}^{\prime}, \ldots, g_{m}^{\prime} \in K\left[x_{1}, \ldots, x_{n}\right]$, and $g_{i}$ is a homogeneous component of $g_{i}^{\prime}$ (i.e., $g_{i}=g_{i_{i}}^{\prime}$ for some $\left.\tau_{i}\right)$. If $h \in \llbracket c \rrbracket_{\mathbf{R e m}, \Gamma}^{\sharp \mathrm{H}}\left(\left\{g_{1}, \ldots, g_{m}\right\}\right)$, then there exists $h^{\prime} \in \llbracket c \rrbracket_{\text {Rem }}^{\sharp}\left(\left\{g_{1}^{\prime}, \ldots, g_{m}^{\prime}\right\}\right)$ such that $h$ is a homogeneous component of $h^{\prime}$. 
Proof. Let us say $G$ is a homogeneous component of $G^{\prime}$ under $\Gamma$ if, for any $p \in G$, there exists $p^{\prime} \in G^{\prime}$ such that $p=p_{\tau}^{\prime}$ for some $\tau$. By induction on $c$, we can prove that if $G$ is a homogeneous component of $G^{\prime}$ under $\Gamma$, then $\llbracket c \rrbracket_{\mathbf{R e m}, \Gamma}^{\sharp \mathrm{H}}(G)$ is a homogeneous component of $\llbracket c \rrbracket_{\operatorname{Rem}, \Gamma}^{\sharp \mathrm{H}}\left(G^{\prime}\right)$ under $\Gamma$.

Theorem 3 (Completeness). Let $g_{i}$ and $g_{i}^{\prime}$ be the same as in Lemma 1. If $\Gamma \vdash c$ and $\llbracket c \rrbracket_{\mathbf{R e m}}^{\sharp}\left(\left\{g_{1}^{\prime}, \ldots, g_{m}^{\prime}\right\}\right)=\{0\}$, then $\llbracket c \rrbracket_{\mathbf{R e m}, \Gamma}^{\sharp \mathrm{H}}\left(\left\{g_{1}, \ldots, g_{m}\right\}\right)=\{0\}$.

Proof. Take $h \in \llbracket c \rrbracket_{\mathbf{R e m}, \Gamma}^{\sharp \mathrm{H}}\left(\left\{g_{1}, \ldots, g_{m}\right\}\right)$. Then there exists $h^{\prime} \in \llbracket c \rrbracket_{\text {Rem }}^{\sharp}\left(\left\{g_{1}^{\prime}\right.\right.$, $\left.\left.\ldots, g_{m}^{\prime}\right\}\right)$ such that $h_{\operatorname{gdeg}(h)}^{\prime}=h$. By assumption, we have $h^{\prime}=0$; therefore $h=0$.

Hence, if $g=0$ is a solution of the postcondition problem, then so is $g^{\prime}=0$ for every homogeneous component $g^{\prime}$ of $g$.

Example 7. Recall Example 3, Theorem 3 and $\llbracket c_{\text {fall }} \rrbracket_{\text {Rem }}^{\sharp}(\{p\})=\{0\}$ guarantee $\llbracket c_{\text {fall }} \rrbracket_{\mathbf{R e m}, \Gamma}^{\sharp \mathrm{H}}\left(\left\{p_{1}\right\}\right)=\{0\}$ and $\llbracket c_{\text {fall }} \rrbracket_{\mathbf{R e m}, \Gamma}^{\sharp \mathrm{H}}\left(\left\{p_{2}\right\}\right)=\{0\}$ since $p_{1}$ and $p_{2}$ are homogeneous components of $p$.

\section{Template-based algorithm}

This section applies our idea to Cachera's template-based invariant-synthesis algorithm [4. We hereafter use metavariable $a$ for a parameter that represents an unknown value. We use metavariable $A$ for a set of parameters. A template on $A$ is an expression of the form $a_{1} p_{1}+\cdots+a_{n} p_{n}$ where $a_{1}, \ldots, a_{n} \in A$; we abuse the metavariable $G$ for a set of templates. We denote the set of templates on $A$ by $T(A)$. A valuation $v$ on $A$ is a map from $A$ to $K$. We can regard $v$ as a map from $T(A)$ to $K\left[x_{1}, \ldots, x_{n}\right]$ by $v\left(a_{1} p_{1}+\cdots+a_{m} p_{m}\right)=v\left(a_{1}\right) p_{1}+\cdots+v\left(a_{m}\right) p_{m}$.

\subsection{Algorithm proposed by Cachera et al.}

Cachera et al. proposed a sound template-based algorithm for the postcondition problem. Their basic idea is to express a fixed point by constraints on the parameters in a template in order to avoid fixed-point iteration.

To recall the algorithm of Cachera et al., we establish several definitions.

Definition 5. An equality constraint on $A$ is an expression of the form $\langle G \equiv$ $\left.G^{\prime}\right\rangle$, where $G, G^{\prime} \subseteq T(A)$. A constraint set on $A$, or simply constraints, is a set of equality constraints on $A$; a constraint set is represented by the metavariable $C$. We may write $(A, C)$ for a constraint set $C$ on $A$ to make $A$ explicit. A valuation $v$ on $A$ satisfies an equality constraint $\left\langle G \equiv G^{\prime}\right\rangle$ on $A$, written $v \models\left\langle G \equiv G^{\prime}\right\rangle$, if $v(G)$ and $v\left(G^{\prime}\right)$ generate the same ideal. A solution of a constraint set $(A, C)$ is a valuation on $A$ that satisfies all constraints in $C$. If $v$ is a solution of $(A, C)$, we write $v \models(A, C)$, or simply $v \models C$. A template $a_{1} p_{1}+\cdots+a_{m} p_{m}$ is a $\mathrm{GH}$ template of g-degree $\tau$ under $\Gamma$ if $p_{1}, \ldots, p_{m}$ are $G H$ polynomials of $g$-degree $\tau$. 


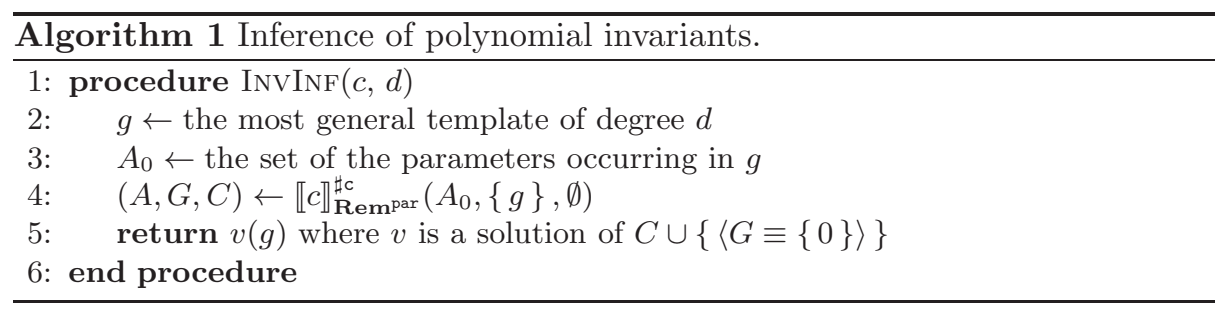

We extend the definition of the remainder computation to operate on templates.

Definition 6. $\mathbf{R e m}^{\mathrm{par}}(A, f, p)$ is a pair $\left(A^{\prime}, f-p q\right)$ where $q$ is the most general template of degree $\operatorname{deg}(f)-\operatorname{deg}(p)$, the parameters of which are fresh; $A^{\prime}$ is the set of the parameters appearing in $q$. We write $\mathbf{R e m}^{\mathrm{par}}\left(A,\left\{p_{1}, \ldots, p_{m}\right\}\right.$, p) for $\left(A^{\prime}, G^{\prime}\right)$, where $\left(A_{i}, r_{i}\right)=\operatorname{Rem}^{\mathrm{par}}\left(A, p_{i}, p\right)$ and $A^{\prime}=\bigcup A_{i}$ and $G^{\prime}=$ $\left\{r_{1}, \ldots, r_{m}\right\}$.

For example, if the set of variables is $\{x\}$, then $\operatorname{Rem}^{\mathrm{par}}\left(\emptyset, x^{2}, x+1\right)=\left(\left\{a_{1}, a_{2}\right\}\right.$, $\left.x^{2}-\left(a_{1} x+a_{2}\right)(x+1)\right)$; the most general template of degree $\operatorname{deg}\left(x^{2}\right)-\operatorname{deg}(x+1)=$ 1 with variable $x$ is $a_{1} x+a_{2}$. By expressing a remainder using a template, we can postpone the choice of a remainder operator to a later stage; for example, if we instantiate $\left(a_{1}, a_{2}\right)$ with $(1,-1)$, then we have the standard remainder operator on $\mathbb{R}[x]$.

We recall the constraint generation algorithm proposed by Cachera et al. We write $\left(A_{i}, G_{i}, C_{i}\right)$ for $\llbracket c_{i} \rrbracket_{\mathbf{R e m}^{\mathrm{par}}}(A, G, C)$ in each case of the following definition.

$$
\begin{aligned}
& \llbracket \mathbf{s k i p} \rrbracket_{\mathbf{R e m}^{\mathrm{par}}}^{\sharp \mathrm{c}}(A, G, C)=(A, G, C) \\
& \llbracket x:=p \rrbracket_{\text {Rem }^{\text {par }}}^{\sharp \mathrm{c}}(A, G, C)=(A, G[x:=p], C) \\
& \llbracket c_{1} ; c_{2} \rrbracket_{\text {Rem }^{\text {par }}}^{\sharp c}(A, G, C)=\llbracket c_{1} \rrbracket_{\text {Rem }^{\mathrm{par}}}^{\sharp \mathrm{c}}\left(\llbracket c_{2} \rrbracket_{\text {Rem }}^{\sharp \mathrm{c}}(A, G, C)\right) \\
& \text { 【if } p=0 \text { then } c_{1} \text { else } c_{2} \rrbracket_{\mathrm{Rem}^{\mathrm{par}}}(A, G, C)=\left(A_{3}, p \cdot G_{2} \cup G_{3}, C_{1} \cup C_{2}\right) \\
& \text { where }\left(A_{3}, G_{3}\right)=\operatorname{Rem}^{\mathrm{par}}\left(A_{1} \cup A_{2}, G_{1}, p\right) \\
& \llbracket \text { while } p \bowtie 0 \text { do } c_{1} \rrbracket_{\operatorname{Rem}^{\mathrm{par}}}^{\sharp \mathrm{c}}(A, G, C)=\left(A_{1}, G, C_{1} \cup\left\{\left\langle G \equiv G_{1}\right\rangle\right\}\right)
\end{aligned}
$$

$\llbracket c \rrbracket_{\text {Rem }^{\text {par }}}^{\sharp c}(A, G, C)$ accumulates the generated parameters to $A$ and the generated constraints to $C$. $A$ is augmented by fresh parameters at the if statement where $\mathbf{R e m}^{\text {par }}$ is called. At a while statement, $\left\langle G \equiv G_{1}\right\rangle$ is added to the constraint set to express the loop-invariant condition.

Algorithm 1 solves the postcondition problem with the constraint-generating subprocedure $\llbracket c \rrbracket_{\mathbf{R e m}^{\mathrm{par}}}^{\sharp c}$. This algorithm, given a program $c$ and degree $d$, returns a set of postconditions that can be expressed by an algebraic condition with degree $d$ or lower. The algorithm generates the most general template $g$ of degree $d$ for the postcondition and applies $\llbracket c \rrbracket_{\text {Rem }^{\text {par }}}^{\sharp c}$ to $g$. For the returned set of polynomials $G$ and the constraint set $C$, the algorithm computes a solution of $C \cup\langle G \equiv\{0\}\rangle$; the equality constraint $\langle G \equiv\{0\}\rangle$ states that $v(g)=0$, where $v$ is a solution of the constraint set $C \cup\langle G \equiv\{0\}\rangle$, has to hold at the end of $c$ regardless of the initial state. 
This algorithm is proved to be sound: If $p \in \operatorname{Invinf}(c, d)$, then $p=0$ holds at the end of $c$ for any initial states [4. Completeness was not mentioned in their paper.

Remark 1. The algorithm requires a solver for the constraints of the form $\langle G \equiv$ $\left.G^{\prime}\right\rangle$. This is the problem of finding $v$ that equates $\langle G\rangle$ and $\left\langle G^{\prime}\right\rangle$; therefore, it can be solved using a solver for the ideal membership problems [5]. To avoid high-cost computation, Cachera et al. proposed heuristics to solve an equality constraint.

Example 8. We explain how $\operatorname{InvINF}\left(c_{\text {fall }}, 3\right)$ works. The algorithm generates a degree-3 template $q\left(x, v, t, x_{0}, v_{0}, t_{0}, a, d t, g, \rho\right)$ over $\left\{x, v, t, x_{0}, v_{0}, t_{0}, a, d t, g, \rho\right\}$. The algorithm then generates the following constraints by $\llbracket c_{\text {fall }} \rrbracket_{\mathrm{Rem}^{\mathrm{par}}}^{\sharp \mathrm{cH}}:\langle\{q(x, v$, $\left.\left.\left.t, x_{0}, v_{0}, t_{0}, a, d t, g, \rho\right)\right\} \equiv\left\{q\left(x+v d t, v-g d t-\rho v d t, t+d t, x_{0}, v_{0}, t_{0}, a, d t, g, \rho\right)\right\}\right\rangle$ (from the body of the loop) and $\left\langle\left\{q\left(x_{0}, v_{0}, t_{0}, x_{0}, v_{0}, t_{0}, a, d t, g, \rho\right)\right\} \equiv\{0\}\right\rangle$. By solving these constraints with a solver for ideal membership problems [5] or with the heuristics proposed by Cachera et al. [4, and by applying the solution to $q\left(x, v, t, x_{0}, v_{0}, t_{0}, a, d t, g, \rho\right)$, we obtain $p$ in Example 2.

\subsection{Restriction to GH templates}

We define a variation $\llbracket c \rrbracket_{\mathbf{R e m}_{T}^{\text {parh }}, \Gamma}^{\sharp c H}$ of the constraint generation algorithm in which we use only GH polynomial templates. $\llbracket c \rrbracket_{\mathbf{R e m}_{\Gamma}^{\text {part }}, \Gamma}^{\sharp c H}$ differs from $\llbracket c \rrbracket_{\mathbf{R e m}^{\text {par }}}^{\sharp c}$ in that it is parameterized also over $\Gamma$, not only over the remainder operation used in the algorithm. The remainder operator $\operatorname{Rem}_{\Gamma}^{\mathrm{parH}} H \Gamma(A, f, p)$ returns a pair $\left(A \cup A^{\prime}, f-p q\right)$ where $q$ is the most general GH template with g-degree $\operatorname{gdeg}(f) \operatorname{gdeg}(p)^{-1}$, with degree $\operatorname{deg}(f)-\operatorname{deg}(p)$, and with fresh parameters; $A^{\prime}$ is the set of the parameters that appear in $q$. $\operatorname{Rem}_{\Gamma}^{\mathrm{parH}}(A, G, p)$ is defined in the same way as Definition 6 for a set $G$ of polynomials. We again write $\left(A_{i}, G_{i}, C_{i}\right)$ for $\llbracket c_{i} \rrbracket_{\mathbf{R e m}_{\Gamma}^{\text {parr }}}^{\sharp \mathrm{c}}(A, G, C)$ in each case of the following definition.

$$
\begin{aligned}
& \llbracket \mathbf{s k i p} \rrbracket_{\operatorname{Rem}_{\Gamma}^{\text {parH }}, \Gamma}^{\text {par }}(A, G, C)=(A, G, C) \\
& \llbracket x:=p \rrbracket_{\operatorname{Rem}_{\Gamma}^{\mathrm{parH}}, \Gamma}^{\sharp \mathrm{cH}}(A, G, C)=(A, G[x:=p], C)
\end{aligned}
$$

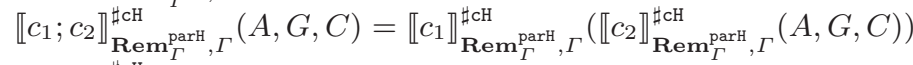

$$
\begin{aligned}
& \text { 【if } p=0 \text { then } c_{1} \text { else } c_{2} \rrbracket_{\operatorname{Rem}_{\Gamma}^{\sharp c \mathrm{cH}}, \Gamma}^{\mathrm{parH}}(A, G, C)=\left(A_{3}, p \cdot G_{2} \cup G_{3}, C_{1} \cup C_{2}\right) \\
& \text { where }\left(A_{3}, G_{3}\right)=\operatorname{Rem}_{\Gamma}^{\mathrm{parH}} H \Gamma\left(A_{1} \cup A_{2}, G_{1}, p\right) \\
& \llbracket \text { while } p \bowtie 0 \text { do } c_{1} \rrbracket_{\operatorname{Rem}_{\Gamma}^{\sharp \mathrm{cH}}, \Gamma}^{\mathrm{parH}}(A, G, C)=\left(A_{1}, G, C_{1} \cup\left\{\left\langle G \equiv G_{1}\right\rangle\right\}\right)
\end{aligned}
$$

Algorithm 2 is a variant of Algorithm 1 in which we restrict a template to GH one.

The algorithm InvINF ${ }^{\mathrm{H}}$ takes the input $\tau$ that specifies the g-degree of the invariant at the end of the program $c$. We have not obtained a theoretical result for $\tau$ to be passed to $\operatorname{INVINF}^{\mathrm{H}}$ so that it generates a good invariant. However, during the experiments in Section 7 , we found that the following strategy often 


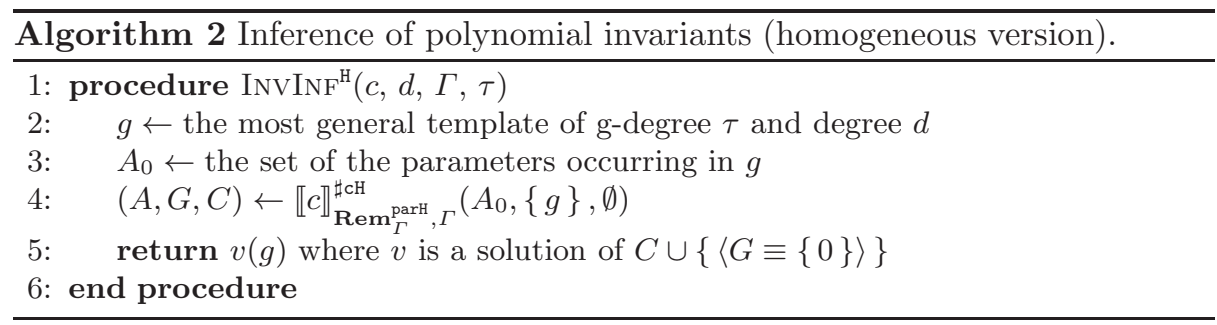

works: Pass the g-degree of the monomial of interest. For example, if we are interested in a property related to $x$, then pass $\Gamma(x)$ (i.e., $L)$ to $\operatorname{INvINF}^{\mathrm{H}}$ for the invariant $-g t^{2}+g t_{0}^{2}-2 t v+2 t_{0} v_{0}+2 x-2 x_{0}=0$. How to help a user to find such "monomial of her interest" is left as an interesting future direction.

The revised version of the invariant inference algorithm is sound; at the point of writing, completeness of $\operatorname{INVINF}^{\mathrm{H}}$ with respect to INVINF is open despite the completeness of $\llbracket c \rrbracket_{\text {Rem }, \Gamma}^{\sharp H}$ with respect to $\llbracket c \rrbracket_{\text {Rem }}^{\sharp}$.

Theorem 4 (Soundness). Suppose $\Gamma \vdash c, d \in \mathbb{N}$, and $\tau \in$ GDeg. Set $P_{1}$ to the set of polynomials that can be returned by $\operatorname{INVINF}^{\mathrm{H}}(c, d, \tau)$; set $P_{2}$ to those by $\operatorname{InvInf}(c, d)$. Then, $P_{1} \subseteq P_{2}$.

\section{Experiment}

We implemented Algorithm 2 and conducted experiments. Our implementation Fastind $d_{\text {dim }}$ takes a program $c$, a maximum degree $d$ of the template $g$ in the algorithm, and a monomial $w$. It conducts type inference of $c$ to generate $\Gamma$ and calls $\operatorname{INVINF}^{\mathrm{H}}\left(c, d, \Gamma\right.$, gdeg $\left.{ }_{\Gamma}(w)\right)$. The type inference algorithm is implemented with OCaml; the other parts (e.g., a solver for ideal-equality constraints) are implemented with Mathematica.

To demonstrate the merit of our approach, we applied this implementation to the benchmark used in the experiment by Cachera et al. 44 and compared our result with that of their implementation, which is called Fastind. The entire experiment was conducted on a MacBook Air 13-inch Mid 2013 model with a 1.7 $\mathrm{GHz}$ Intel Core i7 (with two cores, each of which has $256 \mathrm{~KB}$ of L2 cache) and 8 GB of RAM (1600 MHz DDR3). The modules written in OCaml were compiled with ocamlopt. The version of OCaml is 4.02.1. The version of Mathematica is 10.0.1.0. We refer the reader to [4,18,19] for detailed descriptions of each program in the benchmark. Each program contains a nested loop with a conditional branch (e.g., dijkstra), a sequential composition of loops (e.g., divbin), and nonlinear expressions (e.g., petter (n).) We generated a nonlinear invariant in each program.

Table 1 shows the result. The column deg shows the degree of the generated polynomial, $t_{\text {sol }}$ shows the time spent by the ideal-equality solver (ms), \#m shows the number of monomials in the generated template, $t_{\text {inf }}$ shows the time spent by the dimension-type inference algorithm $(\mathrm{ms})$, and $t_{\text {inf }}+t_{\text {sol }}$ shows the 
sum of $t_{\text {inf }}$ and $t_{\text {sol }}$. By comparing \# $m$ for Fastind with that of Fastind $d_{d i m}$, we can observe the effect of the use of GH polynomials on the template sizes. Comparison of $t_{s o l}$ for Fastind with that of Fastind ${ }_{d i m}$ suggests the effect on the constraint reduction phase; comparison of $t_{s o l}$ for Fastind with $t_{\text {inf }}+t_{\text {sol }}$ for Fastind $_{\text {dim }}$ suggests the overhead incurred by g-degree inference.

Discussion The size of the templates, measured as the number of monomials $(\# m)$, was reduced in 13 out of 20

\begin{tabular}{l|c|c|c|c|c|c|c} 
Name & & \multicolumn{2}{c|}{ Fastind } & \multicolumn{5}{c}{ Fastind } \\
& deg & $t_{\text {sol }}$ & $\# m$ & $t_{\text {inf }}$ & $t_{\text {sol }}$ & $t_{\text {inf }}+t_{\text {sol }}$ & $\# m$ \\
\hline dijkstra & 2 & 9.29 & 21 & 0.456 & 8.83 & 9.29 & 21 \\
divbin & 2 & 0.674 & 21 & 0.388 & 0.362 & 0.750 & 8 \\
freire1 & 2 & 0.267 & 10 & 0.252 & 0.258 & 0.510 & 10 \\
freire2 & 3 & 2.51 & 35 & 0.463 & 2.60 & 3.06 & 35 \\
cohencu & 3 & 1.74 & 35 & 0.434 & 0.668 & 1.10 & 20 \\
fermat & 2 & 0.669 & 21 & 0.583 & 0.669 & 1.25 & 21 \\
wensley & 2 & 104 & 21 & 0.436 & 28.5 & 28.9 & 9 \\
euclidex & 2 & 1.85 & 45 & 1.55 & 1.39 & 2.94 & 36 \\
lcm & 2 & 0.811 & 28 & 0.513 & 0.538 & 1.05 & 21 \\
prod4 & 3 & 31.6 & 84 & 0.149 & 2.78 & 2.93 & 35 \\
knuth & 3 & 137 & 220 & 4.59 & 136 & 141 & 220 \\
mannadiv & 2 & 0.749 & 21 & 0.515 & 0.700 & 1.22 & 18 \\
petter1 & 2 & 0.132 & 6 & 0.200 & 0.132 & 0.332 & 6 \\
petter2 & 3 & 0.520 & 20 & 0.226 & 0.278 & 0.504 & 6 \\
petter3 & 4 & 1.56 & 35 & 0.226 & 0.279 & 0.505 & 7 \\
petter4 & 5 & 7.15 & 56 & 0.240 & 0.441 & 0.681 & 8 \\
petter5 & 6 & 17.2 & 84 & 0.228 & 0.326 & 0.554 & 9 \\
petter10 & 11 & 485 & 364 & 0.225 & 0.354 & 0.579 & 14 \\
sumpower1 & 3 & 2.20 & 35 & 0.489 & 2.31 & 2.80 & 35 \\
sumpower5 & 7 & 670 & 330 & 0.469 & 89.1 & 89.6 & 140 \\
\hline
\end{tabular}
programs by using $\mathrm{GH}$ polynomials. The value of $t_{\text {sol }}$ decreased for these 13 programs; it is almost the same for the other programs. \# $m$ did not decrease for the other seven programs because the extension of the type inference procedure mentioned above introduced useless auxiliary variables. We expect that such variables can be eliminated by using a more elaborate program analysis.

By comparing $t_{s o l}$ for Fastind and

Table 1. Experimental result. $t_{\text {inf }}+t_{\text {sol }}$ for Fastind dim $_{\text {, }}$, we can observe that the inference of the gdegree assignment sometimes incurs an overhead for the entire execution time if the template generated by Fastind is sufficiently small; therefore, Fastind is already efficient. However, this overhead is compensated in the programs for which Fastind requires more computation time.

To summarize, our current approach is especially effective for a program for which (1) the existing invariant-synthesis algorithm is less efficient owing to the large size of the template and (2) a nontrivial g-degree assignment can be inferred. We expect that our approach will be effective for a wider range of programs if we find a more competent g-degree inference algorithm.

\section{Related work}

The template-based algebraic invariant synthesis proposed to date [4, 20, has focused on reducing the problem to constraint solving and solving the generated constraints efficiently; strategies for generating a template have not been the main issue. A popular strategy for template synthesis is to iteratively increase the degree of a template. This strategy suffers from an increase in the size of a template in the iterations when the degree is high.

Our claim is that prior analysis of a program effectively reduces the size of a template; we used the dimension type system for this purpose in this paper inspired by the principle of quantity dimensions in the area of physics. Of course, 
there is a tradeoff between the cost of the analysis and its effect on the templatesize reduction; our experiments suggest that the cost of dimension type inference is reasonable.

Semialgebraic invariants (i.e., invariants written using inequalities on polynomials) are often useful for program verification. The template-based approach is also popular in semialgebraic invariant synthesis. One popular strategy in template-based semialgebraic invariant synthesis is to reduce this problem to one of semidefinite programming, for which many efficient solvers are widely available.

As of this writing, it is an open problem whether our idea regarding GH polynomials also applies to semialgebraic invariant synthesis; for physically meaningful programs, at least, we guess that it is reasonable to use GH polynomials because of the success of the quantity dimension principle in the area of physics. A possible approach to this problem would be to investigate the relationship between GH polynomials and Stengle's Postivstellensatz [22, which is the theoretical foundation of the semidefinite-programming approach mentioned above. There is a homogeneous version of the Stengle's Positivstellensatz 8, Theorem II.2]; because the notion of homogeneity considered there is equivalent to generalized homogeneity introduced in this paper, we conjecture that this theorem provides a theoretical foundation of an approach to semialgebraic invariant synthesis using GH polynomials.

Although the application of the quantity dimension principle to program verification is novel, this principle has been a handy tool for discovering hidden knowledge about a physical system. A well-known example in the field of hydrodynamics is the motion of a fluid in a pipe [2]. One fundamental result in this regard is that of Buckingham [3], who stated that any physically meaningful relationship among $n$ quantities can be rewritten as one among $n-r$ independent dimensionless quantities, where $r$ is the number of the quantities of the base dimension. Investigating the implications of this theorem in the context of our work is an important direction for future work.

The term "generalized homogeneity" appears in various areas; according to Hankey et al. [10, a function $f\left(x_{1}, \ldots, x_{n}\right)$ is said to be generalized homogeneous if there are $a_{1}, \ldots, a_{n}$ and $a_{f}$ such that, for any positive $\lambda, f\left(\lambda^{a_{1}} x_{1}, \ldots\right.$, $\left.\lambda^{a_{n}} x_{n}\right)=\lambda^{a_{f}} f\left(x_{1}, \ldots, x_{n}\right)$. Barenblatt 2 points out that the essence of the quantity dimension principle is generalized homogeneity. Although we believe our GH polynomials are related to the standard definition, we have not fully investigated the relationship at the time of writing.

Our idea (and the quantity dimension principle) seems to be related to invariant theory [17 in mathematics. Invariant theory studies various mathematical structures using invariant polynomials. A well-known fact is that a ring of invariants is generated by homogeneous polynomials [5, Chapter 7]; GH polynomials can be seen as a generalization of the notion of degree.

The structure of $K\left[x_{1}, \ldots, x_{n}\right]$ resulting from the notion of the generalized degrees is an instance of graded rings from ring theory. Concretely, $R$ is said to be graded over an Abelian group $\mathbb{G}$ if $R$ is decomposed into the direct sum of a family 
of additive subgroups $\left\{R_{g} \mid g \in \mathbb{G}\right\}$ and these subgroups satisfy $R_{g} \cdot R_{h} \subseteq R_{g h}$ for all $g, h \in \mathbb{G}$. Then, an element $x \in R$ is said to be homogeneous of degree $g$ if $x \in R_{g}$. We leave an investigation of how our method can be viewed in this abstract setting as future work.

\section{Conclusion}

We presented a technique to reduce the size of a template used in template-based invariant-synthesis algorithms. Our technique is based on the finding that, if an algebraic invariant of a program $c$ exists, then there is a GH invariant of $c$; hence, we can reduce the size of a template by synthesizing only a GH polynomial. We presented the theoretical development as a modification of the framework proposed by Cachera et al. and empirically confirmed the effect of our approach using the benchmark used by Cachera et al. Although we used the framework of Cachera et al. as a baseline, we believe that we can apply our idea to the other template-based methods [1,4,7, 16, 19, 21.

Our motivation behind the current work is safety verification of hybrid systems, in which the template method is a popular strategy. For example, Gulwani et al. 9] proposed a method of reducing the safety condition of a hybrid system to constraints on the parameters of a template by using Lie derivatives. We expect our idea to be useful for expediting these verification procedures.

In this regard, Suenaga et al. [11, 23, 24, have recently proposed a framework called nonstandard static analysis, in which one models the continuous behavior of a system as an imperative or a stream-processing program using an infinitesimal value. An advantage of modeling in this framework is that we can apply program verification tools without an extension for dealing with continuous dynamics. However, their approach requires highly nonlinear invariants for verification. This makes it difficult to apply existing tools, which do not handle nonlinear expressions well. We expect that the current technique will address this difficulty with their framework.

We are also interested in applying our idea to decision procedures and satisfiability modulo theories (SMT) solvers. Support of nonlinear predicates is an emerging trend in many SMT solvers (e.g., Z3 [15]). Dai et al. 6] proposed an algorithm for generating a semialgebraic Craig interpolant using semidefinite programming [6]. Application of our approach to these method is an interesting direction for future work.

\section{Acknowledgment}

We appreciate annonymous reviewers, Toshimitsu Ushio, Naoki Kobayashi and Atsushi Igarashi for their comments. This work is partially supported by JST PRESTO, JST CREST, KAKENHI 70633692, and in collaboration with the Toyota Motor Corporation. 


\section{References}

1. Adjé, A., Garoche, P., Magron, V.: Property-based polynomial invariant generation using sums-of-squares optimization. In: Blazy, S., Jensen, T. (eds.) SAS 2015. LNCS, vol. 9291, pp. 235-251. Springer (2015)

2. Barenblatt, G.I.: Scaling, self-similarity, and intermediate asymptotics: dimensional analysis and intermediate asymptotics, vol. 14. Cambridge University Press (1996)

3. Buckingham, E.: On physically similar systems; illustrations of the use of dimensional equations. Phys. Rev. 4, 345-376 (Oct 1914)

4. Cachera, D., Jensen, T.P., Jobin, A., Kirchner, F.: Inference of polynomial invariants for imperative programs: A farewell to Gröbner bases. Sci. Comput. Program. 93, 89-109 (2014)

5. Cox, D.A., Little, J., O'Shea, D.: Ideals, Varieties, and Algorithms: An Introduction to Computational Algebraic Geometry and Commutative Algebra, 3/e (Undergraduate Texts in Mathematics). Springer-Verlag New York, Inc., Secaucus, NJ, USA (2007)

6. Dai, L., Xia, B., Zhan, N.: Generating non-linear interpolants by semidefinite programming. In: Sharygina, N., Veith, H. (eds.) CAV 2013. LNCS, vol. 8044, pp. 364-380. Springer (2013)

7. Garg, P., Löding, C., Madhusudan, P., Neider, D.: ICE: A robust framework for learning invariants. In: Biere, A., Bloem, R. (eds.) CAV 2014. LNCS, vol. 8559, pp. 69-87. Springer (2014)

8. Gonzalez-Vega, L., Lombardi, H.: Smooth parametrizations for several cases of the Positivstellensatz. Mathematische Zeitschrift 225(3), 427-451 (1997), http://dx.doi.org/10.1007/PL00004620

9. Gulwani, S., Tiwari, A.: Constraint-based approach for analysis of hybrid systems. In: Gupta, A., Malik, S. (eds.) CAV 2008. LNCS, vol. 5123, pp. 190-203. Springer (2008)

10. Hankey, A., Stanley, H.E.: Systematic application of generalized homogeneous functions to static scaling, dynamic scaling, and universality. Physical Review B 6(9), 3515 (1972)

11. Hasuo, I., Suenaga, K.: Exercises in nonstandard static analysis of hybrid systems. In: Madhusudan, P., Seshia, S.A. (eds.) CAV 2012. LNCS, vol. 7358, pp. 462-478. Springer (2012)

12. Kennedy, A.: Dimension types. In: ESOP'94. pp. 348-362 (1994)

13. Kennedy, A.: Programming Languages and Dimensions. Ph.D. thesis, St. Catharine's College (Mar 1996)

14. Lankford, D., Butler, G., Brady, B.: Abelian group unification algorithms for elementary terms. Contemporary Mathematics 29, 193-199 (1984)

15. de Moura, L.M., Bjørner, N.: Z3: an efficient SMT solver. In: TACAS 2008. pp. 337-340 (2008)

16. Müller-Olm, M., Seidl, H.: Computing polynomial program invariants. Inf. Process. Lett. 91(5), 233-244 (2004)

17. Neusel, M.D.: Invariant theory. The American Mathematical Society (2000)

18. Rodríguez-Carbonell, E.: Some programs that need polynomial invariants in order to be verified. http://www.cs.upc.edu/ erodri/webpage/polynomial_invariants/list.html (Accessed on January 25th, 2016)

19. Rodríguez-Carbonell, E., Kapur, D.: Generating all polynomial invariants in simple loops. J. Symb. Comput. 42(4), 443-476 (2007) 
20. Sankaranarayanan, S., Sipma, H., Manna, Z.: Non-linear loop invariant generation using Gröbner bases. In: POPL 2004. pp. 318-329 (2004)

21. Somenzi, F., Bradley, A.R.: IC3: where monolithic and incremental meet. In: FMCAD 2011. pp. 3-8 (2011)

22. Stengle, G.: A nullstellensatz and a positivstellensatz in semialgebraic geometry. Mathematische Annalen 207(2), 87-97 (1974)

23. Suenaga, K., Hasuo, I.: Programming with infinitesimals: A while-language for hybrid system modeling. In: Aceto, L., Henzinger, M., Sgall, J. (eds.) ICALP 2011. LNCS, vol. 6756, pp. 392-403. Springer (2011)

24. Suenaga, K., Sekine, H., Hasuo, I.: Hyperstream processing systems: nonstandard modeling of continuous-time signals. In: Giacobazzi, R., Cousot, R. (eds.) POPL 2013. pp. 417-430. ACM (2013)

\section{A Proof of Theorem 4}

To prove Theorem 4 we define renaming of parameters and constraints.

Definition 7. For an injection $\iota: A \rightarrow A^{\prime}$, we write $\iota:(A, G, C) \preceq\left(A^{\prime}, G^{\prime}\right.$, $\left.C^{\prime}\right)$ if $G^{\prime}=\iota^{*}(G)$ and $C^{\prime}=\iota^{*}(C)$ where $\iota^{*}$ maps $a^{\prime} \in \iota(A)$ to $\iota^{-1}\left(a^{\prime}\right)$ and $a^{\prime} \in \iota\left(A^{\prime} \backslash \iota(A)\right)$ to 0 .

The injection $\iota$ gives a renaming of parameters. The relation $\iota:(A, G$, $C) \preceq\left(A^{\prime}, G^{\prime}, C^{\prime}\right)$ reads $G$ and $C$ are obtained from $G^{\prime}$ and $C^{\prime}$ by renaming the parameters in $\iota(A)$ using $\iota$ and substituting 0 to those not in $\iota(A)$.

Lemma 2. If $\iota:(A, G, C) \preceq\left(A^{\prime}, G^{\prime}, C^{\prime}\right)$, then there exists $\kappa$ such that (1) $\kappa: \llbracket c \rrbracket_{\mathbf{R e m}^{\mathrm{par}}, \Gamma}^{\sharp c \mathrm{H}}(A, G, C) \preceq \llbracket c \rrbracket_{\mathbf{R e m}^{\mathrm{par}}}^{\sharp \mathrm{c}}\left(A^{\prime}, G^{\prime}, C^{\prime}\right)$ and (2) $\kappa$ is an extension of $\iota$.

Proof. Induction on the structure of $c$.

Proof of Theorem 4 Let $g \in T\left(A_{0}\right)$ be the most general template of generalized degree $\tau$ and degree $d$ and $g^{\prime} \in T\left(A_{0}^{\prime}\right)$ be the most general template of degree $d$. Without loss of generality, we assume $A_{0} \subseteq A_{0}^{\prime}$ and $g^{\prime}=g+g_{1}$ for some $g_{1} \in T\left(A_{0}^{\prime} \backslash A_{0}\right)$. Let $(A, G, C)=\llbracket c \rrbracket_{\operatorname{Rem}^{\mathrm{par}}, \Gamma}^{\sharp c \mathrm{H}}\left(A_{0},\{g\}, \emptyset\right)$ and $\left(A^{\prime}, G^{\prime}\right.$, $\left.C^{\prime}\right)=\llbracket c \rrbracket_{\text {Rem }}^{\sharp \mathrm{par}}\left(A_{0}^{\prime},\left\{g^{\prime}\right\}, \emptyset\right)$. Then, from Lemma 2] there exists $\kappa$ such that $\kappa:(A, G, C) \preceq\left(A^{\prime}, G^{\prime}, C^{\prime}\right)$ and $\kappa$ is an extension of the inclusion mapping $\iota: A_{0} \rightarrow A_{0}^{\prime}$. Suppose $v(g)$ is a result of $\operatorname{INVINF}^{\mathrm{H}}(c, d, \tau)$ where $v$ is a solution to $C \cup\{\langle G \equiv\{0\}\rangle\}$. Define a valuation $v^{\prime}$ on $A^{\prime}$ by

$$
v^{\prime}\left(a^{\prime}\right)= \begin{cases}v(a) & a^{\prime}=\kappa(a) \text { for some } a \in A \\ 0 & \text { Otherwise }\end{cases}
$$

Then, $v^{\prime}\left(g^{\prime}\right)=v^{\prime}\left(g+g_{1}\right)=v^{\prime}(g)$; the second equation holds because $v^{\prime}\left(a^{\prime}\right)$ is constantly 0 on any $a^{\prime} \in A^{\prime} \backslash A$. All the parameters in $g$ are in $A_{0}$ and $\kappa$ is an identity on $A_{0}$. Therefore, $v^{\prime}(g)=v(g)$. It suffices to show that $v^{\prime} \models$ $C^{\prime} \cup\left\{\left\langle G^{\prime} \equiv\{0\}\right\rangle\right\}$, which indeed holds from the definition of $v^{\prime}$ since $v \models$ $C \cup\{\langle G \equiv\{0\}\rangle\}$ and $C$ and $G$ are renaming of $C^{\prime}$ and $G^{\prime}$. 